\title{
Maps of Ionospheric F2-Layer Characteristics Derived from GPS Radio Occultation Observations
}

\author{
Lung-Chih Tsai ${ }^{1,2, *}$, Chao-Han Liu ${ }^{2,3}$, Tung-Yuan Hsiao ${ }^{4}$, and Chia-Chia Chang ${ }^{3}$ \\ ${ }^{1}$ Center for Space and Remote Sensing Research, National Central University, Chung-Li, Taiwan, ROC \\ ${ }^{2}$ Graduate Institute of Space Science, National Central University, Chung-Li, Taiwan, ROC \\ ${ }^{3}$ Institute of Astronomy and Astrophysics, Academia Sinica, Taipei, Taiwan, ROC \\ ${ }^{4}$ Department of Information Technology, Hsing Wu College, Taipei, Taiwan, ROC
}

Received 30 January 2008, accepted 7 July 2008

\begin{abstract}
The Global Positioning System (GPS) radio occultation (RO) technique has been used to receive multi-channel GPS carrier phase signals from low Earth orbiting (LEO) satellites and demonstrate active limb sounding of the Earth's ionosphere. Applying Abel inversion through compensated total electron content (TEC) values, the GPS RO observations can obtain ionospheric electron density $\left(n_{e}\right)$ profiles and then scale F2-layer characteristics including $f o F 2$ and $h m F 2$, especially, $h m F 2$ that cannot be directly deduced from ionosonde observations. From the GPS/MET and FS3/COSMIC missions, we can collect on average two hundred and eighteen hundred vertical $n_{e}$ profiles, respectively, within one day. The retrieved foF2 and $h m F 2$ results have been used to produce numerical maps representing complex properties on a world-wide scale. This paper presents a physically appealing representation of $f_{o F} 2$ and $h m F 2$ medians based on GPS RO data. The derived numerical maps have also been examined by ground-based ionosonde data.
\end{abstract}

Key words: Ionosphere, Electron density, Mapping, Radio occultation, GPS

Citation: Tsai, L. C., C. H. Liu, T. Y. Hsiao, and C. C. Chang, 2009: Maps of ionospheric F2-layer characteristics derived from GPS radio occultation observations. Terr. Atmos. Ocean. Sci., 20, 535-546, doi: 10.3319/TAO.2008.07.07.02(AA)

\section{INTRODUCTION}

The ionosphere is that region of the earth's atmosphere in which free ions and electrons exist in sufficient abundance to affect the properties of electromagnetic waves that are propagated within and through it. The structure of the ionosphere is highly variable. The F2 region is the strongest ionospheric region, and it is also the most variable in time and in space. For the most used empirical ionosphere model, the International Reference Ionosphere (IRI) model (Bilitza 2001), models of the F2-layer critical frequency ( $f F 2)$ and the peak density height $(h m F 2)$ are expressed in terms of elementary functions of latitude, longitude and universal time, multiplied by appropriate coefficients (Rush et al. 1984; URSI 1987) that have been determined by applications of spherical harmonic analyses on the ionosonde data. These analyses are referred to as numerical mapping of $f_{o} F 2$ and $h m F 2$. It is based on vertical-incidence ionosonde observa-

\footnotetext{
* Corresponding author

E-mail:lctsai@csrsr.ncu.edu.tw
}

tions from $\sim 150$ stations throughout the world and theoretically generated $f_{o} F 2$ and $h m F 2$ values to improve representations over ocean and other inaccessible areas. Furthermore, $h m F 2$ values cannot be directly deduced from ionosonde observations and then used in corresponding numerical mapping. For an assumption of a simple ionospheric layer, in which the variation of $n_{e}$ with height is parabolic with plasma frequency, the virtual height at a plasma frequency of $0.834 f o F 2$ in the absence of the Earth's magnetic field equals $h m F 2$ and can be used to estimate $h m F 2$. Other techniques for estimating $h m F 2$ can be carried out by the conversion of a plasma frequency versus virtual height curve to an $n_{e}$ true height profile, i.e., true height analyses, or determined by empirical equations with the transmission factor $M(3000) F 2$ and a correction $(\Delta M)$ to $M(3000) F 2$, formulated in terms of the ratio $f_{o} F 2 / f_{o} E$ (Dudeney 1983) as used in the IRI model. We suggest that physically appealing representations of $f o F 2$ and $h m F 2$ are possible using GPS RO data. An advantage is data compilation includ- 
ing areas over oceans and the southern hemisphere, where few ionosonde stations are positioned. The purpose of this article is to describe $f_{o F} 2$ and $h m F 2$ mapping using GPS $\mathrm{RO}$ observations and to investigate the possibility of incorporating RO data in future numerical coefficients. To map foF 2 and $h m F 2$ parameters, we first discuss the achievable accuracy of input RO data. In sections 3 and 4, spherical harmonic analysis and optimization of $f o F 2$ and $h m F 2$ numerical coefficients are discussed. Throughout GPS/MET and FormoSat3/Constellation Observing System for Meteorology Ionosphere and Climate (FS3/COSMIC) data, the corresponding mapping results of spherical harmonic analysis are further discussed in sections 5 and 6, respectively.

\section{IONOSPHERIC SOUNDING EXPERIMENTS FROM GPS/MET AND FS3/COSMIC}

Probing Earth's atmosphere and ionosphere via GPS RO observations from a LEO satellite was proposed by Jet Propulsion Laboratory in 1988 but was first implemented by the University Corporation for Atmospheric Research (UCAR) (Zou et al. 1995; Ware et al. 1996; Kursinski et al. 1997; Rocken et al. 1997). UCAR launched the MicroLab-1 satellite at a nearly circular orbit of $\sim 735 \mathrm{~km}$ altitude and $\sim 70^{\circ}$ inclination angle in 1995 . The main objectives of this GPS-to-LEO RO mission were numerical weather prediction and long-term monitoring of the Earth's climate and thus it was termed the GPS/Meteorology (GPS/MET) program. Besides which, GPS/MET has also been able to sound the ionosphere from its orbit altitude to the Earth's surface and retrieve one-dimensional profiles of ionospheric $n_{e}$ from measurements of ray-path bending angle or TEC (Hajj and Romans 1998; Hocke and Igarashi 2002a). In the GPS/MET mission, the Microlab-1 spacecraft was equipped with a single antenna that tracked the occulted GPS satellites of setting (or rising) behind the Earth's ionosphere at an angle less than $\sim 60$ degrees between the occultation plane and the LEO orbital plane. A GPS receiver onboard the MicroLab-1 satellite provides up to 250 useful RO observations per day based on 24 GPS satellites. After the GPS/MET mission, further missions flown with GPS RO receivers onboard include the Danish Ørsted, the German CHAMP, the Argentinean SAC-C, the American-German GRACE, the South African SUNSAT, and the Ionosphere Occultation Experiment (IOX) onboard the PICOSAT satellite. After those, the Taiwan FS3/COSMIC satellites were successfully launched on 15 April 2006. In relation to the GPS/MET mission, the FS3/ COSMIC project placed six micro-satellites into different orbits at $\sim 800 \mathrm{~km}$ altitudes, and each micro-satellite was equipped with two high-gain occultation antennas to track both rising and setting GPS satellites. RO measurements on board FS3/COSMIC will cover the entire global atmosphere and ionosphere, providing over 2500 sounding data per day.
When receiving multi-channel GPS carrier phase signals from LEO satellites, an occultation takes place when a GPS satellite sets or rises behind the Earth's ionosphere and lower atmosphere as seen by a receiver in LEO. Each occultation therefore consists of a set of limb-viewing links with ray perigee ranging from the orbiting LEO satellite to the surface of the Earth. $n_{e}$ profiles can be derived, in general, by Abel inversion through the computation of calibrated TEC values under the assumptions of locally spherical symmetry and straight-line propagation. There are several studies examining and estimating the accuracy of retrieved GPS/MET $n_{e}$ profiles obtained with Abel inversions by comparing them with vertical ionosonde data (Hajj and Romans 1998; Schreiner et al. 1999; Tsai et al. 2001). All studies concluded satisfactory peak densities retrieved from RO observations with fractional root-mean-square $(\mathrm{rms})$ differences from $10 \%$ to $20 \%$ in $f_{o} F 2$. Without additional data interpretation the retrieved $n_{e} \mathrm{~s}$ actually suffer from inherent horizontal averaging in Earth angle. To improve spatial resolution and accuracy Straus (1999) and Hajj et al. (2000) have presented a constrained-gradient Abel inversion method, which uses earlier simulated horizontal gradients from other ancillary data (in-situ plasma density, nadir-viewing extreme ultraviolet airglow, and vertical TEC maps derived from ground-based GPS receivers) and constrains the practical Abel inversion considering the horizontal asymmetry effect on $n_{e}$ retrieval. Hocke and Igarashi (2002b) also proposed a two-dimensional recovery method, which can compute electron density within a meridian plane at all latitudes and from the top layer of the LEO satellite altitude to the base of the ionosphere. Meanwhile, both Hernandez-Pajares et al. (2000) and Garcia-Fernandez et al. (2003) have also developed modified Abel inversion algorithms expressing the three-dimensional retrieved $n_{e}$ as the product of a vertical TEC map and a shape function in altitude that provided better statistics than the traditional Abel inversion assuming spherical symmetry. Another improved scheme was also proposed by Tsai and Tsai (2004) and Tsai et al. (2009). The effect of large-scale horizontal gradient in $n_{e}$ was taken into consideration by introducing a compensation procedure for measured TEC values through several close-up RO observations. The TECs could be "compensated" by the ray integral of $n_{e}$ differences between the spherical symmetry $n_{e}$ and the interpolated two-dimensional $n_{e}$ from a set of close-up profiles retrieved from earlier inversion. Applying the compensated TECs to the Abel inversion again and again could reduce error from the spherical symmetry assumption and approach a reliable result. Our investigation showed improved agreement with the ionosonde data when the grouped RO observation number is larger than 3. From several hundred thousand matches within the FS3/COSMIC intense observation period experiment, evaluation of the FS3/COSMIC retrieved $f o F 2 \mathrm{~s}$ through the improved Abel inversion scheme (after two iterations) on compensated TECs is shown 
in Fig. 1. Results of underestimated and overestimated $f_{o} F 2 \mathrm{~s}$ are obtained in high $f_{0} F 2$ values (from 4.5 to $9 \mathrm{MHz}$ ) and low foF 2 values (from 2 to $4 \mathrm{MHz}$ ), respectively, but the fractional mean and $r m s$ foF2 differences are significantly improved. The average $r m s$ foF 2 differences between the ionosonde measurements and the FS3/COSMIC retrievals are improved from 1.67 to $1.07 \mathrm{MHz}$.

As described ionosonde observations cannot provide $h m F 2$ directly and be used to verify retrieved GPS RO $h m F 2$ values. In this paper, we have simulated path TECs by integrating the IRI-modeled $n_{e}$ s. $n_{e}$ profiles can then be retrieved by our improved Abel inversion scheme through simulated TECs. Such simulation is called a "reference" GPS RO experiment in this paper. The retrieved $n_{e}$ profiles can then be compared and verified by the "true" IRI-modeled ionosphere. Figure 2 illustrates a scatter plot and the corresponding least-squares fitting line for the retrieved GPS RO $\mathrm{hmF} 2$ values versus the "true" $h m F 2$ values based on the IRI model. The results have a high linear correlation coefficient of 0.98 and a fitting line with a slope of 0.98 and a positive bias of $5.50 \mathrm{~km}$. The corresponding average mean and $r m s ~ h m F 2$ differences are -2.62 and $6.59 \mathrm{~km}$, respectively, which is about the same as typical range resolution scales of ionosonde sounding.

\section{SPHERICAL HARMONIC ANALYSIS OF IONOSPHERIC CHARACTERISTICS MAPPING}

The construction of a particular solution of the Laplace's partial differential equation on the surface of a sphere (assumed independent of time) is known as spherical surface harmonics. The numerical map for $f o F 2$ or $h m F 2$ can denote a spherical harmonic function, $\Gamma(\theta, \phi)$, in spherical coordinates of $0^{\circ} \leq \theta \leq 180^{\circ}$ and $0^{\circ} \leq \phi \leq 360^{\circ}$, and the spherical surface Laplace equation is:

$\frac{1}{\sin \theta} \frac{\partial}{\partial \theta}\left(\sin \theta \frac{\partial \Gamma}{\partial \theta}\right)+\frac{1}{\sin ^{2} \theta} \frac{\partial^{2} \Gamma}{\partial \phi^{2}}=0$

where $\theta$ is the geographic latitude added by $90^{\circ}, \phi$ could be the geographic longitude (in the Earth coordination) or the local time angle (in the local time system coordination) measured westward from apparent noon (i.e., $0^{\circ}$ denotes noon, and $180^{\circ}$ denotes midnight). The resulting real functions obtained on separation of variables and the GramSchmidt orthogonalization process (Davis 1989) are defined by:

$$
U_{n m}(\theta, \phi)=\sqrt{\frac{2 n+1}{2 \pi} \frac{(n-m) !}{(n+m) !}} P_{n}^{m}(\cos \theta) \cos m \phi
$$

$V_{n m}(\theta, \phi)=\sqrt{\frac{2 n+1}{2 \pi} \frac{(n-m) !}{(n+m) !}} P_{n}^{m}(\cos \theta) \sin m \phi$

$(n=0,1,2, \ldots ; m=0,1,2, \ldots, n)$,

where

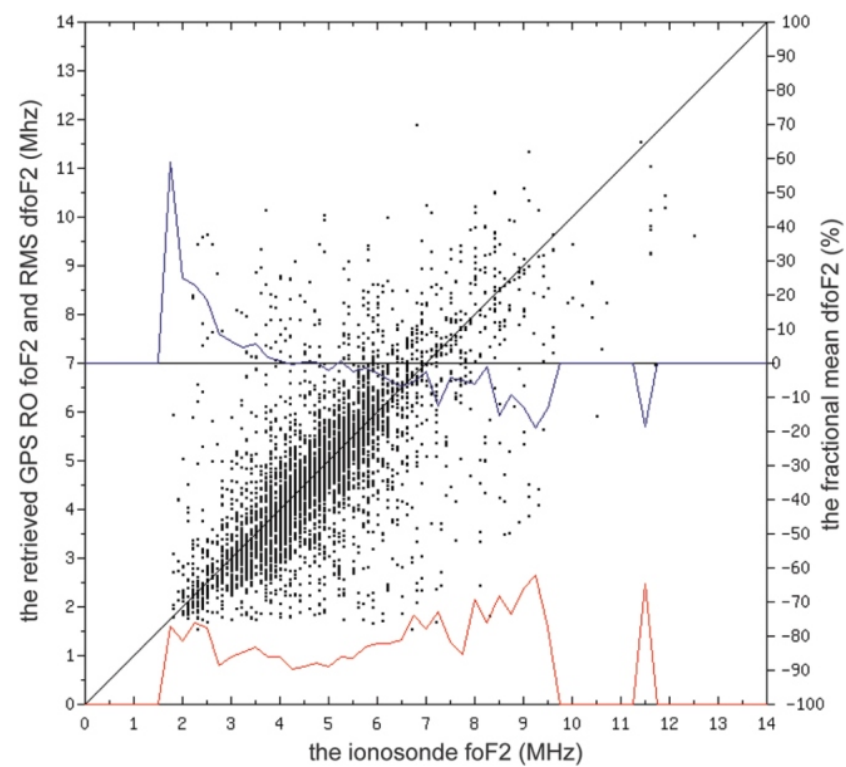

Fig. 1. A scatter plot of the FS3/COSMIC retrieved $f o F 2$ s versus the scaled $f o F 2$ from ionosonde data, their $r m s$ foF 2 difference curve in red (refer to the left $\mathrm{y}$-axis in $\mathrm{MHz}$ ), and their fractional mean $f_{o} F 2$ difference curve in blue (refer to the right $y$-axis in percentage) as a function of the ionosonde $f \circ F 2$.

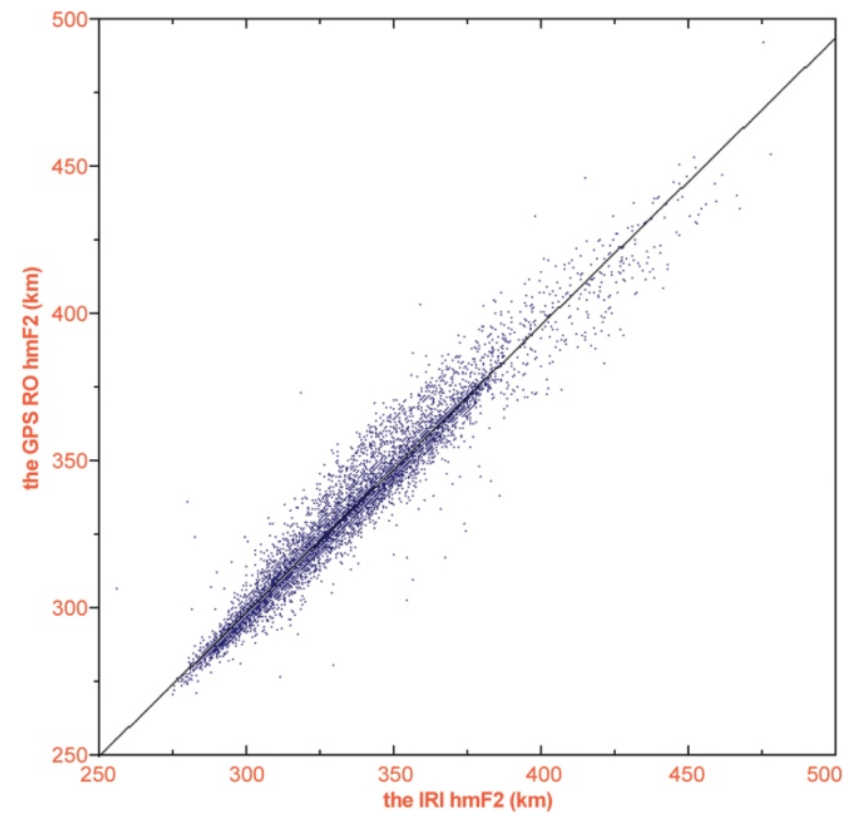

Fig. 2. A scatter plot of the retrieved GPS RO $h m F 2$ s from a reference GPS RO experiment versus the true $h m F 2$ s from the IRI model. 


$$
\begin{aligned}
& P_{n}^{m}(\cos \theta)=\frac{1}{2^{n}} \sin ^{m} \theta \sum_{l=0}^{\left[\frac{n-m}{2}\right]}(-1)^{l} m !\left(\begin{array}{c}
n-2 l \\
m
\end{array}\right) \\
& \left(\begin{array}{l}
n \\
l
\end{array}\right)\left(\begin{array}{c}
2 n-2 l \\
n
\end{array}\right) \cos ^{n-m-2 l} \theta
\end{aligned}
$$

is the familiar associated Legendre polynomial of the first kind of degree $\mathrm{n}$ and order $\mathrm{m}$. The above explicit expression for the associated Legendre function is extracted by the authors. The geographic variation of $f o F 2$ or $h m F 2$ can thus be represented by a series of functions, $U_{n m}(\theta, \phi)$ and $V_{n m}(\theta, \phi)$, analogous to the Earth surface. Notice that the functions $U_{n m}(\theta, \phi)$ and $V_{n m}(\theta, \phi)$ are orthogonal and normal sets in terms of the inner product. There are exactly $2 n$ +1 linearly independent spherical surface harmonics of degree $\mathrm{n}$; these functions are called tesseral spherical harmonics for $m<n$ and sectorial spherical harmonics for $m=$ $\mathrm{n}$. In theory, any continuous function on the surface of a sphere can be uniformly approximated there by means of these orthogonal spherical harmonics with any desired degree of accuracy. In this study, we will choose as our coordinate functions a particular set of the spherical surface harmonics $U_{n m}(\theta, \phi)$ and $V_{n m}(\theta, \phi)$ in the first three orders of $m=0,1$, and 2 only but an optimum degree for each order to separate noise from real longitudinal variation. The determination of the optimum order and degree numbers will be discussed in details within the next section. The spherical harmonics in the order of $m=0,1$, and 2 are respectively specified for terms involving the main latitudinal trend without longitudinal variation, tesseral harmonics $(m=1)$ for including first order longitudinal variation, and second order sectorial and tesseral order harmonics $(m=2)$ for including second order longitudinal variation. The optimum number of terms for $f o F 2$ and $h m F 2$ geographic variation in each order is made by truncating high spectrum harmonics based on a least-square fitting. It is assumed that the values $q_{0}, q_{1}$, and $q_{2}$ are the highest powers of $\cos \theta$ for each of the three orders, and the values $k_{0}, k_{1}$, and $k_{2}$ could be specified as the term index $k$ at the end of each order and are related to the $q$-values as follows:

$k_{0}=q_{0}+1$, and

$k_{r}=k_{r-1}+2\left(q_{r}+1\right)$ for $r=1,2$

A least-square representation in the form of a linear combination of $U_{n m}(\theta, \phi)$ and $V_{n m}(\theta, \phi)$ has many properties of a spherical harmonic analysis. For example, it is periodic in latitude and longitude; the first and second order sectorial and tesseral harmonics are linear combinations of the first and second order longitude variation terms which are weighted according to latitude by the functions $\sin \theta$ and $\sin ^{2} \theta$, respectively. Meanwhile, the fitting of polynomials in $\cos \theta$ has the effect of pulling the data symmetrically away from the equator toward the poles, and the data becomes more uniformly distributed in $\cos \theta$ than in $\theta$ (or the latitude). Furthermore, at high degrees the powers in $\theta$ have much larger absolute values and thus the polynomials in $\theta$ become more wildly fluctuating (i.e., unstable) near the poles than near the equator. In contrast, the polynomials in $\cos \theta$ can also minimize the maximum error and are as stable at the poles as they are at the equator. Figure 3 firstly shows an example representation of $f_{O F} 2$ main latitude variation for the $13^{\text {th }}$ prime GPS/MET daytime data using least-square fitting polynomials in both $\theta$ (red line) and $\cos \theta$ (blue line) of degree 12. It was found that the latitudinal variation could be represented in more detail by the polynomial in $\cos \theta$ than in $\theta$ of the same degree. Moreover, more stable representations near the poles were obtained by using the polynomial in $\cos \theta$, too. More examples representing higher order longitudinal variation will be discussed in the following sections.

\section{OPTIMUM SEPARATION OF NOISE}

In this paper, input ionospheric data ( $f o F 2$ and $h m F 2$ ) are affected by noise produced from a number of sources including limitations of equipments, retrieval techniques such as the Abel inversion method, and intrinsic random fluctuations of the physical phenomena being measured. Generally, the values of noise are small compared to the main physical variations; they are assumed to be distributed independently and normally with mean zero and standard deviation $\sigma$. Thus a certain amount of smoothing is necessary for numerical mapping, but its effect must be carefully studied since we wish to represent the physical characteristic with as much detail as possible. The method employed here is based on residuals between the $f_{o F}$ or $h m F 2$ values being fitted and the fitting values from a smoothing (or filtering) process by truncating a series of spherical surface functions, $U_{n m}(\theta, \phi)$ and $V_{n m}(\theta, \phi)$, described in the previous section. A standard deviation $\mathrm{e}_{\mathrm{K}}$ of the residuals can be defined as following:

$$
\begin{aligned}
& E_{K}=\sum_{i=1}^{N}\left\{y_{i}-\sum_{m=0}^{2} \sum_{n=0}^{q_{m}}\left[A_{n m} U_{n m}\left(\theta_{i}, \phi_{i}\right)+B_{n m} V_{n m}\left(\theta_{i}, \phi_{i}\right)\right]\right\}^{2}, \\
& \text { and } e_{K}^{2}=\frac{E_{K}}{N-K-1} \\
& {\left[K=k_{2}=q_{0}+1+2\left(q_{1}+1\right)+2\left(q_{2}+1\right)\right],}
\end{aligned}
$$

where $E_{K}$ is the sum of squares of residuals at a term number of $K$ (or $k_{2}$ defined in the last section), $N$ is the number of $f \circ F 2$ or $h m F 2$ measurements, $y_{i}$, (being also the RO observation number), and $(N-K-1)$ is the number of degrees of freedom remaining after subtraction of one degree for each term in the series $U_{n m}(\theta, \phi)$ and $V_{n m}(\theta, \phi)$. At specified order and degree numbers for the spherical surface functions the coefficients $A_{n m}$ and $B_{n m}$ can be determined by the 


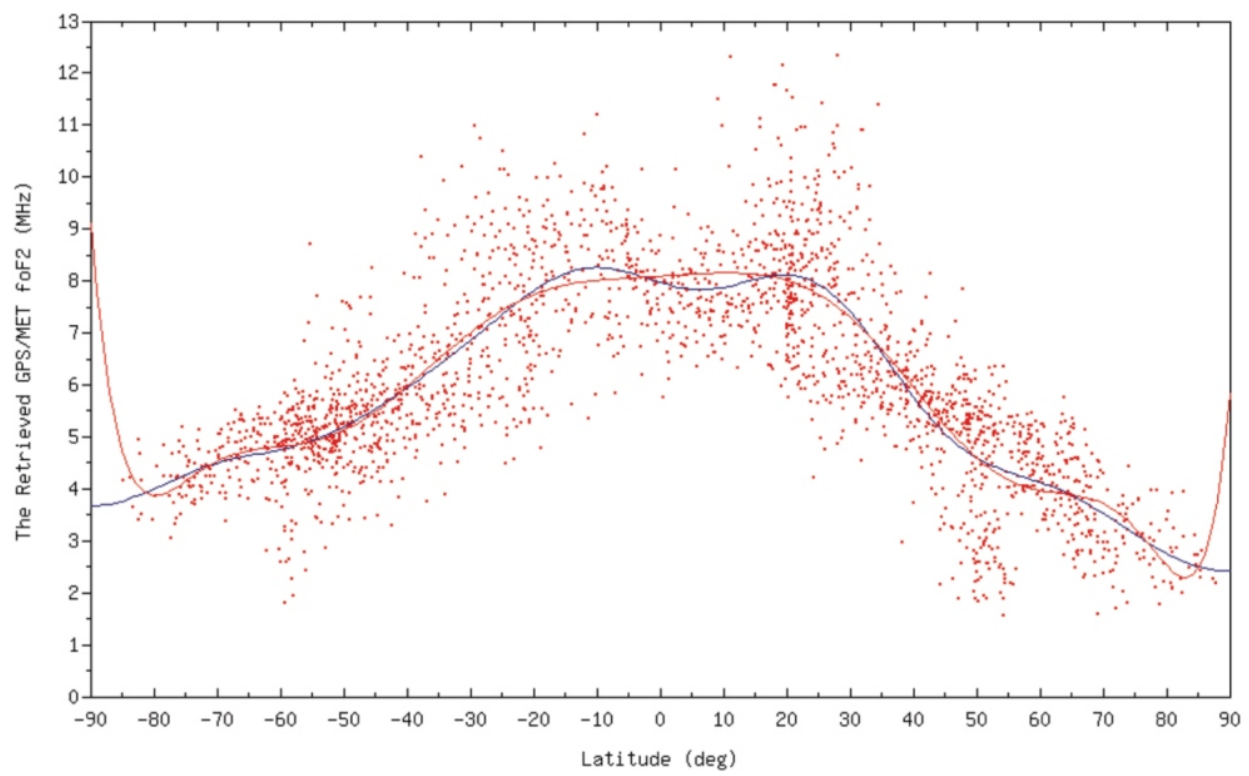

Fig. 3. An example representation of $f o F 2$ main latitude variation for the $13^{\text {th }}$ prime GPS/MET daytime data using least-square fitting polynomials of degree 12 in both latitude $\theta$ (red line) and $\cos \theta$ (blue line).

method of least squares. The residuals in Eq. (6) are modeling residuals, which are not equal to input data errors from retrieving or measurements. Residuals can approach zero as the number $\mathrm{K}$ of terms in the series increases. However, we know the retrieved $f_{0} F 2 \mathrm{~s}$ and $h m F 2 \mathrm{~s}$ are affected by noise; hence zero residuals are not desired. In the least squares analysis under each order, the values $e_{K}$ usually decrease at first at a rate dependent upon the smoothness of $\Gamma(\theta, \phi)$, taper off, and cease to diminish appreciably after a certain point of cutoff. Our problem is therefore to give more objects and quantitative estimation of the noise for its optimum separation from real physical variation by means of mathematical data analyses. The smoothing (filtering) in the geographic dimensions is performed by determining the best term indexes $k_{0}, k_{1}$, and $k_{2}$ (or the degree indexes $q_{0}$, $q_{1}$, and $q_{2}$ ) for the orthogonal series for foF2 and $h m F 2$ mapping. The sum of coefficient squares is convergent, therefore, a necessary condition for the convergence of a series is that the general term approaches zero. It is shown (Davis 1989) that if the numerical map $\Gamma(\theta, \phi)$ has a continuous derivature of $\alpha^{\text {th }}$ order, an absolute amplitude $\mathrm{C}_{\mathrm{nm}}$ can be defined as $\left(\mathrm{A}_{\mathrm{nm}}{ }^{2}+\mathrm{B}_{\mathrm{nm}}{ }^{2}\right)^{1 / 2}$ and approach zero at least as fast as $\mathrm{n}^{-\alpha}$. The criterion adopted for determining the optimum cutoff is therefore to search for a tendency to level off the absolute amplitude. When $\mathrm{C}_{\mathrm{nm}}$ is plotted against $\mathrm{n}$ in a log-log scale, the curve defined by smoothing the points on the graph will have a slope less than or equal to $-\alpha$. It is safe to assume that the geographic variation of $f_{o F} 2$ or $h m F 2$ has at least a piecewise continuous first derivative. Thus the slope of its log-log relationship between the squared amplitude and the degree number should be -1 or less. To illustrate the method, each of the thirteen prime time data of the GPS/MET mission has been divided into eight parts each of which is of a 45-degree geomagnetic longitude range and mostly has more than $400 \mathrm{RO}$ observations. Spherical harmonic analysis was performed separately for each part. Figure 4 shows the average $C_{n m}$ against the degree number $n$ in a log-log scale at each of the first four orders (blue line for the zero-order analysis, cyan line for the first order analysis, red line for the second order analysis, and brown line for the third order analysis). It is shown that the smoothed slopes of $\mathrm{C}_{\mathrm{nm}}$ for the first three order analyses (not including the third order analysis) appear to be less than -1 but larger than -2 in agreement with the theory. In the zero-order analysis the graph of $\mathrm{C}_{\mathrm{nm}}$ against $\mathrm{n}$ shows a tendency to level off around degree 24 , giving the optimum cutoff $q_{0}$. In a similar manner we determine the optimum cutoffs to be degrees 20 and 15 for the first and second order terms $q_{1}$ and $q_{2}$ in longitude.

\section{5. foF2 AND $h m F 2$ MAPPING RESULTS OF THE GPS/MET MISSION}

In this study, an improved Abel inversion scheme has been applied to GPS/MET RO observations and more than forty thousand ionospheric $n_{e}$ profiles and corresponding foF 2 and $h m F 2$ values within 13 prime times ( 250 days) were collected from April 1995 to February 1997. There are on average about thirty-five hundred RO observations recorded within a GPS/MET prime time (about twenty days). The beginning and ending days and the numbers of recorded RO observations for all primes are shown in the first four columns of Table 1. In the following, foF 2 and $h m F 2$ mapping functions have been developed with a total term 


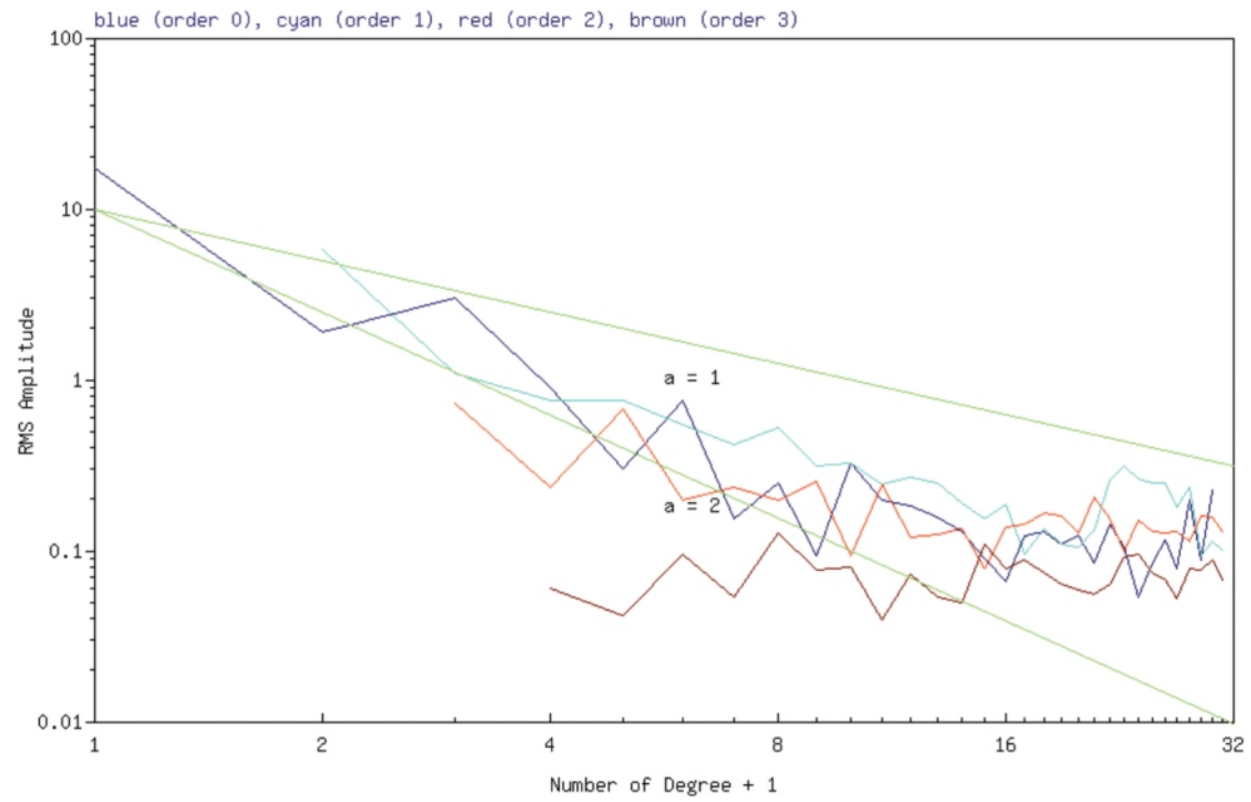

Fig. 4. Graphs of the average amplitude versus the degree number at the $0^{\text {th }}$ (in blue), $1^{\text {st }}$ (in cyan), $2^{\text {nd }}$ (in red), and $3^{\text {rd }}$ (in brown) group order. The a $=1$ and $\mathrm{a}=2$ lines (in green) show the slopes of its log-log relationship to be -1 and -2 , representing a piecewise continuous first and second derivative map, respectively.

Table 1. Estimates of standard deviation error for a numerical mapping foF2 representation from GPS/MET observations.

\begin{tabular}{lccccccccccccc}
\hline Prime \# & $\mathbf{1}$ & $\mathbf{2}$ & $\mathbf{3}$ & $\mathbf{4}$ & $\mathbf{5}$ & $\mathbf{6}$ & $\mathbf{7}$ & $\mathbf{8}$ & $\mathbf{9}$ & $\mathbf{1 0}$ & $\mathbf{1 1}$ & $\mathbf{1 2}$ & $\mathbf{1 3}$ \\
\hline Beginning day & $95 \%$ & $95 \%$ & $95 \%$ & $95 \%$ & $95 \%$ & $96 \%$ & $96 \%$ & $96 \%$ & $96 \%$ & $96 \%$ & $96 \%$ & $96 \%$ & $97 \%$ \\
& 108 & 170 & 223 & 283 & 328 & 022 & 076 & 128 & 186 & 237 & 293 & 350 & 033 \\
Ending day & $95 \%$ & $95 \%$ & $95 \%$ & $95 \%$ & $95 \%$ & $96 \%$ & $96 \%$ & $96 \%$ & $96 \%$ & $96 \%$ & $96 \%$ & $97 \%$ & $97 \%$ \\
& 130 & 192 & 242 & 305 & 349 & 042 & 097 & 148 & 207 & 258 & 312 & 005 & 054 \\
Number of occultation & 1275 & 4158 & 3265 & 4160 & 3008 & 3296 & 3923 & 3180 & 3812 & 3552 & 2777 & 4274 & 4766 \\
A/S status & OFF & OFF & ON & OFF & ON & ON & ON & ON & ON & ON & ON & ON & OFF \\
$\begin{array}{l}\text { Frequencies of Kp } \\
\text { indices (> 3) }\end{array}$ & 72 & 25 & 23 & 49 & 24 & 19 & 38 & 6 & 4 & 40 & 33 & 6 & 33 \\
$\begin{array}{l}\text { Standard deviation } \\
\text { (MHz) of foF2 fitting }\end{array}$ & 1.02 & 0.77 & 0.84 & 1.09 & 0.92 & 0.89 & 0.98 & 0.77 & 0.72 & 0.88 & 1.11 & 0.97 & 0.90 \\
\hline
\end{tabular}

number, $\mathrm{K}$, of $99\left(q_{0}=24, q_{1}=20\right.$, and $\left.q_{2}=15\right)$. These functions $\Gamma(\lambda, \theta)$ therefore can represent the continuous time variations of an ionospheric F2-layer critical frequency characteristic on a world-wide basis and can be used to compute its value at any desired location and instant in time. Such functions are referred to as numerical maps. In this study, mostly more than four hundred foF 2 and $h m F 2$ values have been used to determine a series of spherical surface functions to produce a numerical map for each prime time and every 45-degree geomagnetic longitude range. As described the geographic variation of retrieved $f_{o} F 2$ and $h \mathrm{mF} 2$ values can thus be represented by a series of spherical harmonic functions analogous to the Earth surface in the lati- tude and local time system coordination. Example results of mixed latitudinal and longitudinal foF 2 and $h m F 2$ variation are illustrated by the two-dimensional images of Figs. 5 and 6 , respectively, using the $13^{\text {th }}$ prime time GPS/MET data at the geomagnetic longitude ranges of $90^{\circ} \sim 135^{\circ}$. The solid points from Figs. 5 and 6 denote the line-of-viewing tangent point locations at the $\mathrm{F} 2$ peak of $\mathrm{RO}$ observations. The local time angle $0^{\circ}$ denotes noon and $180^{\circ}$ denotes midnight. As pointed out in section 2, the Microlab-1 spacecraft of the GPS/MET mission was equipped with a single antenna that tracked the occulted GPS satellites in view of setting (or rising for a few percentages) behind the Earth's ionosphere at an angle less than $\sim 60$ degrees of the occultation plane out of 


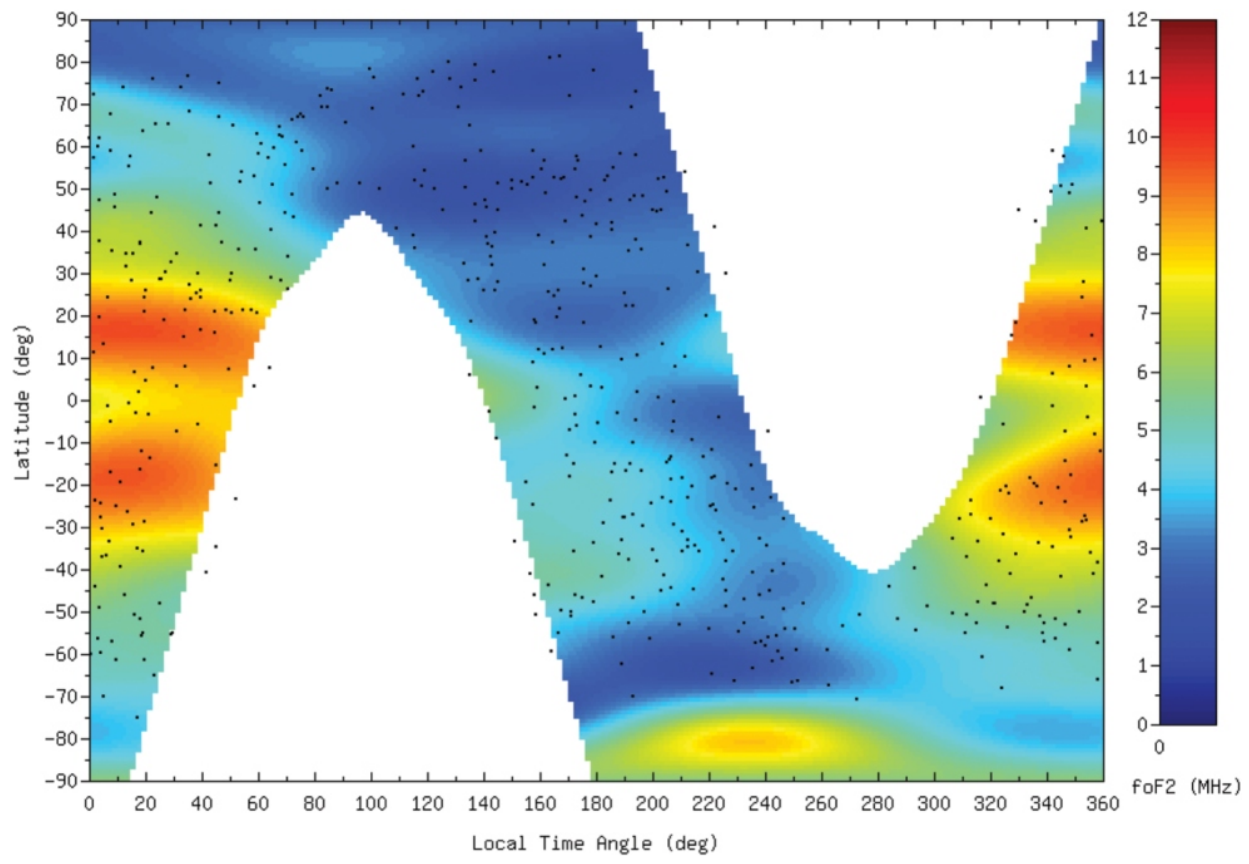

Fig. 5. Mixed latitude and second order longitude trend $f \circ F 2$ mapping using the GPS/MET $13^{\text {th }}$ prime time data at $90^{\circ} \sim 135^{\circ}$ geomagnetic longitudes.

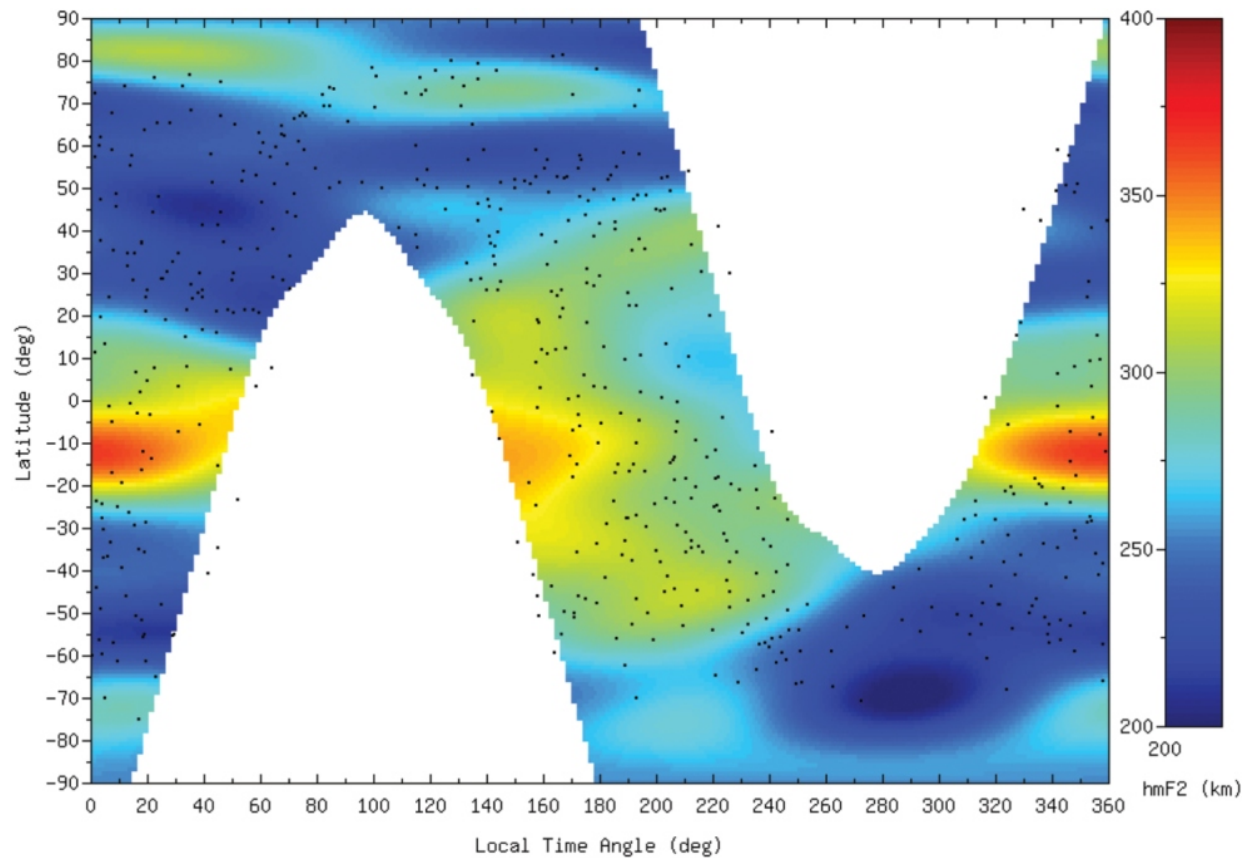

Fig. 6. Mixed latitude and second order longitude trend $h m F 2$ mapping using the GPS/MET $13^{\text {th }}$ prime time data at $90^{\circ} \sim 135^{\circ}$ geomagnetic longitudes.

the LEO orbital plane. Since the MicroLab-1 satellite was located at a nearly circular orbit of $\sim 735 \mathrm{~km}$ altitude and $\sim 70^{\circ}$ inclination angle to receive GPS signals, the GPS RO observations are irregularly distributed onto part of the Earth surface which covers most of the latitudes betweens the poles but only $\sim 30$ longitudinal degrees from both sides of the LEO orbit. Therefore, to obtain an "effective" representation of the geographic $f_{o F} 2$ and $h m F 2$ variation, we just consider the colored areas where GPS RO observations were available as shown from Figs. 5 and 6. In the regions of low-latitude and daytime (the local time angle $<100^{\circ}$ or $>300^{\circ}$ ) as shown in Fig. 5, the $f o F 2 \mathrm{~s}$ are clearly higher than 
the values in high-latitude and/or recorded at nighttime. Furthermore, for both images of Figs. 5 and 6, we note that, in the dayside (the local time angle $<100^{\circ}$ or $>300^{\circ}$ ) equatorial and low-latitude region, features of the equatorial anomaly were observed during the local time period from 8 am to $6 \mathrm{pm}$.

To further evaluate the goodness of the fits of spherical surface function coefficients, the rows from $2^{\text {nd }}$ to $7^{\text {th }}$ in Table 1 list the beginning and ending days, the numbers of recorded RO observations, the GPS A/S status of each prime time, the analyzed standard deviation of $f o F 2$ mapping, and the analyzed standard deviation of $h m F 2$ mapping. As discussed, the noise (or error) of representation of F2-layer characteristic will be studied in terms of residuals obtained from subtracting numerical map values from corresponding RO data. The residuals might be due to random deviations caused by noise inherent in ionospheric data and small systematic variations that have not been represented. As shown in Table 1, the resulting standard deviation of residuals from the mapping analysis of $\mathrm{RO}$ foF 2 values remains fairly stable at less than $0.86 \mathrm{MHz}$ within the GPS/MET mission and has a mean value of $0.74 \mathrm{MHz}$. The standard deviation values of different prime times are not related to the numbers of RO observations and nor GPS A/S status. It can be seen that the $f_{o} F 2$ values determined by the numerical mapping yield smaller residuals, for examples of: $0.66,0.68$, 0.64 , and $0.62 \mathrm{MHz}$ for the $2^{\text {nd }}, 3^{\text {rd }}, 8^{\text {th }}$, and $9^{\text {th }}$ prime data analyses, respectively, in the middle of the years than at the beginning (or end) of the years. Meanwhile, Fig. 7 shows the standard deviation values (refer to the right y-axis) in solid points and the profile of monthly frequencies (refer to the left $\mathrm{y}$-axis) of geomagnetic $\mathrm{Kp}$ index values larger than 2 . $\mathrm{Kp}$ indices are based on the range of variation within a 3 -hour period of the day observed from about a dozen selected magnetic observations and serve a practical function as the basis of geophysical activity on a scale from 0 (for 'very quiet') to 9 (for 'very disturbed'). We note there are comparative low frequencies of Kp larger than 2 at the $2^{\text {nd }}$, $3^{\text {rd }}, 8^{\text {th }}$, and $9^{\text {th }}$ prime times of GPS/MET when the numerical mapping of $f_{o} F 2$ also yields smaller residuals.

\section{6. $f \circ F 2$ AND $h m F 2$ MAPPING RESULTS BASED ON THE FS3/COSMIC DATA}

In the FS3/COSMIC mission, all six spacecraft (FM1 to FM6) were integrated and launched together into a parking orbit of $515 \mathrm{~km}$ altitude; whereupon each spacecraft was separated and then transferred from the parking orbits to their final orbits at $\sim 800 \mathrm{~km}$. Figure 8 shows daily orbit ascending angles of the six FS3/COSMIC spacecraft. Similar orbit ascending angles for all six spacecraft were presented at the beginning of FS3/COSMIC mission; FM5 was transferred firstly onto the targeted orbit, and then FM2, FM6, FM4, FM3, and FM1 finally. The final spacecraft orbits are separated into 30 degrees and the daily RO measurements on board FS3/COSMIC are distributed uniformly onto the entire atmosphere and/or ionosphere after 2007. A comprehensive view of the numbers of RO measurements on board FS3/COSMIC and successfully retrieved $n_{e}$ profiles from each spacecraft is shown in Fig. 9. Since 28 July 2006, raw excess phase data and the retrieved $n_{e}$ profiles are available from the Taiwan Analysis Center for COSMIC

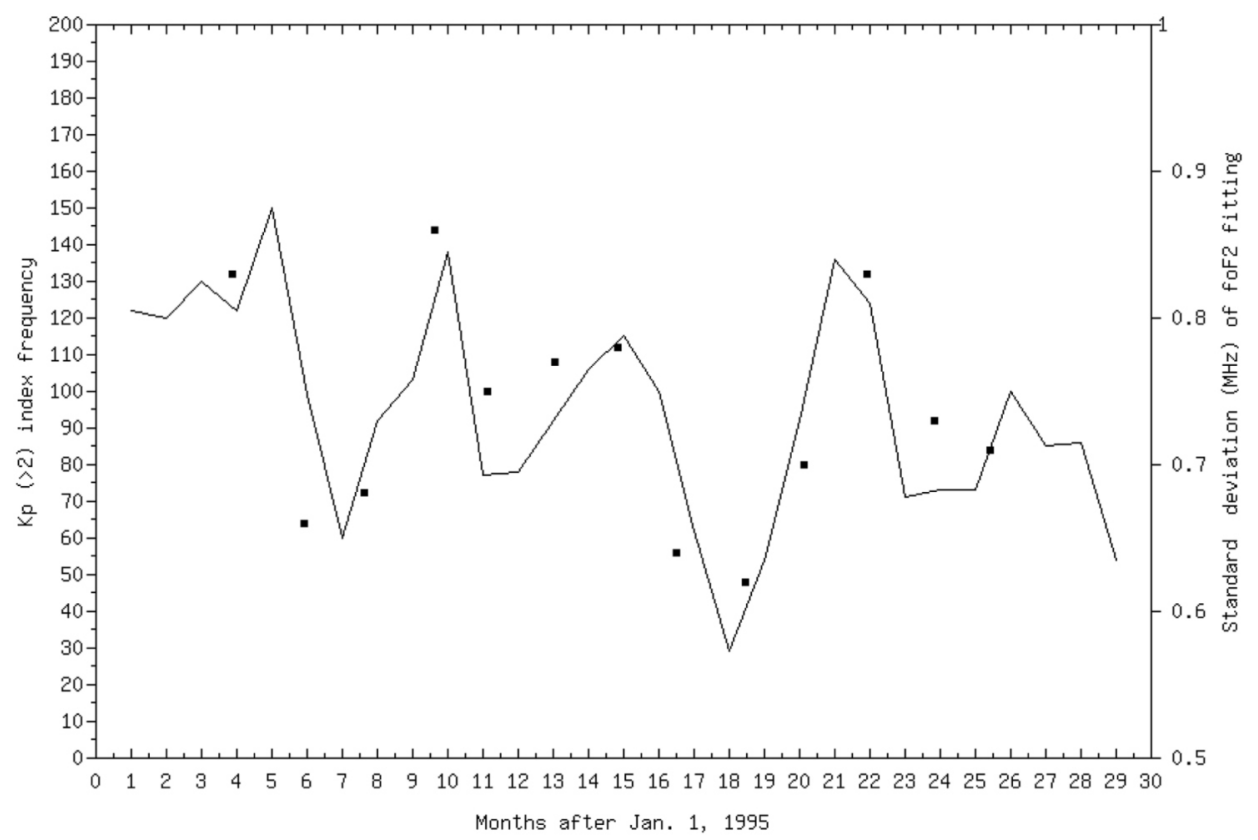

Fig. 7. The comparison between the standard deviation of $f o F 2$ mapping and the monthly frequencies of geomagnetic Kp index values larger than 2. 


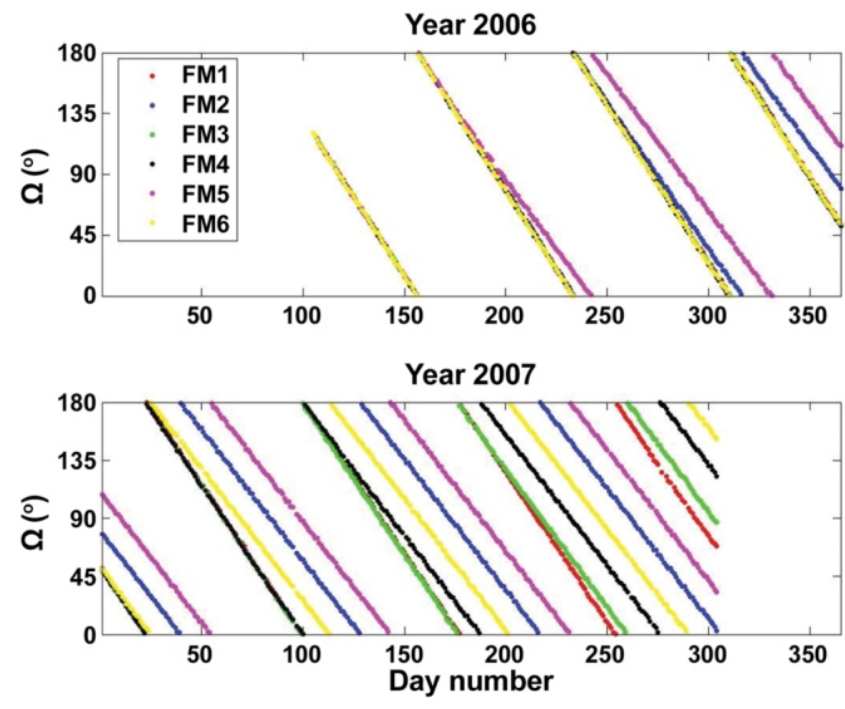

Fig. 8. Daily orbit ascending angles of the six FS3/COSMIC spacecraft.
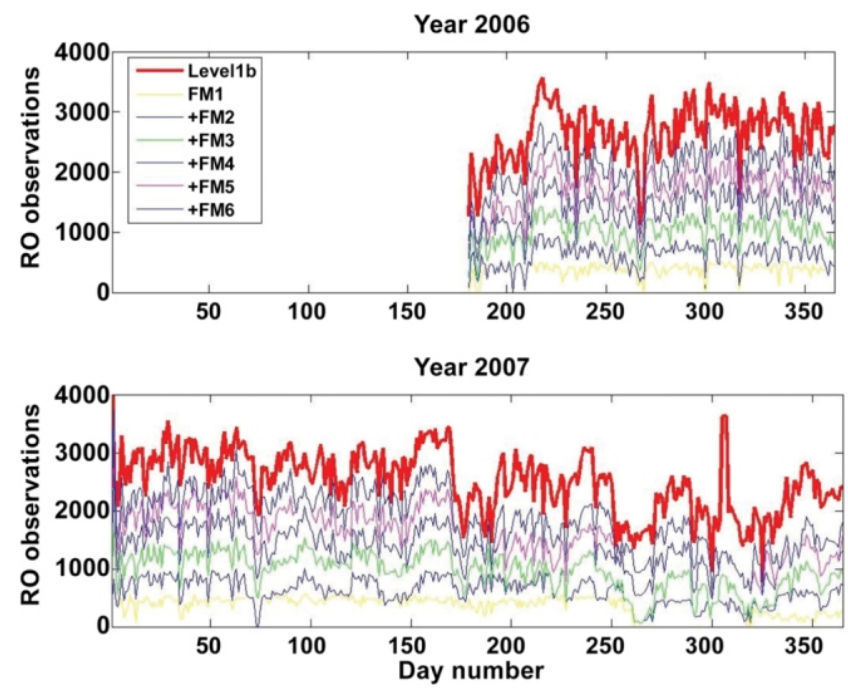

Fig. 9. A comprehensive view of GPS RO measurements (in red) on board FS3/COSMIC and the accumulated numbers of $n_{e}$ profiles successfully retrieved and summed up from FM1, FM2, FM3, FM4, FM5, and then FM6.

(TACC, http://tacc.cwb.gov.tw/en/) and the COSMIC Data Analysis and Archive Center (CDACC, http://www.cosmic .ucar.edu/cdacc/). Generally FS3/COSMIC can perform over $2500 \mathrm{RO}$ measurements per day, and more than $70 \%$ of the $\mathrm{RO}$ measurements can be successfully retrieved into $n_{e}$ profiles. For the remaining RO measurements the atmospheric excess phase calibration failed and the $n_{e}$ profiles could not be obtained. The failed measurements were usually caused by locking onto GPS carrier signals either starting too late or ending too early.

As described, there are on the average about eighteen hundred $n_{e}$ profiles and corresponding $f o F 2$ and $h m F 2$ re- trieved per day within the FS3/COSMIC mission. In the following, we have applied spherical harmonics analyses on monthly foF2 and $h m F 2$ values at geographic coordinates and within every local time (LT) hour. Example results of $f o F 2$ and $h m F 2$ numerical maps are illustrated by the two-dimensional images in Figs. 10 and 11, respectively, using the FS3/COSMIC data in August, 2007. From Figs. 10 and 11, the solid points denote the line-of-viewing tangent point locations at the F2 peak of RO observations, and three curves in black present positions with magnetic dip latitudes of $+30^{\circ}, 0^{\circ}$, and $-30^{\circ}$ from the top down. The top-left, top-right, bottom-left, and bottom-right images in Fig. 10 (Fig. 11) represent the 2007 August $f_{0} F 2(\mathrm{hmF2})$ numerical map at: 0200, 0800, 1400, and 2000 LT, respectively. From Fig. 10 the highest-latitude area in the south is in continual darkness and has lower $n_{e}$ than the highest-latitude area at the north that is in continual daylight because its summer. Features of the diurnal ionosphere variations are obtained and illustrated as follows. The $f o F 2 \mathrm{~s}$ are greater by day than by night; $f_{0} F 2$ increases promptly after sunrise and continues to increase for a few hours after noon and then decreases. There is a general tendency for $h m F 2$ to fall at dawn and then rise during the afternoon or evening. In low latitudes and at the equator, $h m F 2$ reaches a high level by about 1400 LT and then falls such that by late at night it is about $100 \mathrm{~km}$ lower than in the afternoon, and the F2 layer is much thicker near the equator than elsewhere particularly in the daytime. Furthermore, we note that, in equatorial and low-latitude regions, features of the equatorial anomaly are observed in the daytime, and two foF 2 crests lie along the $+30^{\circ}$ and $-30^{\circ}$ magnetic dip latitudes. The features are developed after sunrise and become strongest at about 1400 LT. It is well known that equatorial anomaly results are something of the equatorial fountain feature and are caused by an eastward electric field at the magnetic equator creating a steady upward $\boldsymbol{E} \times \boldsymbol{B} / B^{2}$ plasma drift. The drift raises plasmas from the equator across magnetic field lines to higher altitudes. After losing momentum, the electrons slide down, assisted by gravity, along the field lines to either side of the equator to form two crests. This is called the fountain effect (Hanson and Moffett 1966). One important factor is that ionization changes due to $\boldsymbol{E} \times \boldsymbol{B}$ drift are particularly matched to magnetic dip latitude and northsouth asymmetry effects; these are incorporated and shown in Figs. 10 and 11, too. Theoretically, in the absence of neutral winds, the upward $\boldsymbol{E} \times \boldsymbol{B}$ drift produces almost identical effects at conjugate points; however, neutral winds can cause conjugate-hemisphere differences by modulating the fountain and moving ionospheric electrons at the conjugate hemispheres to different altitudes. Complete mapping images and modeling coefficients based on FS3/COSMIC data can be found from "http://140.115.111.228/", hosted by the Center for Space and Remote Sensing Research, National Central University, and are organized in exactly the same form as in section 4 . 

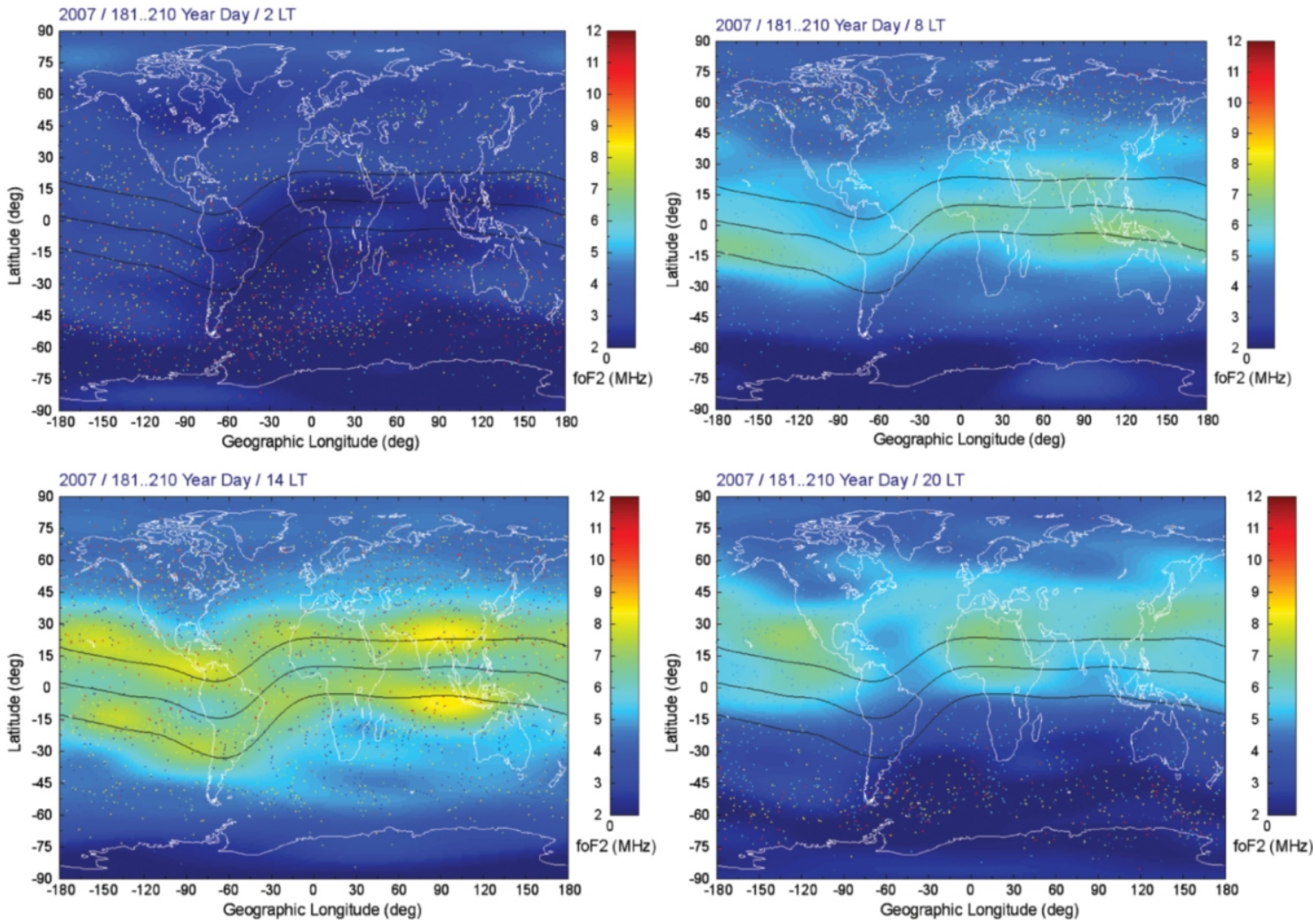

Fig. 10. The 2007 August $f o F 2$ numerical maps at 0200, 0800, 1400, and 2000 LT are assigned to the top-left, top-right, bottom-left, and bottom-right images, respectively. The solid points denote the line-of-viewing tangent point locations at the F2 peak of RO observations, and three curves in black present positions with magnetic dip latitudes of $+30^{\circ}, 0^{\circ}$, and $-30^{\circ}$ from the top down.
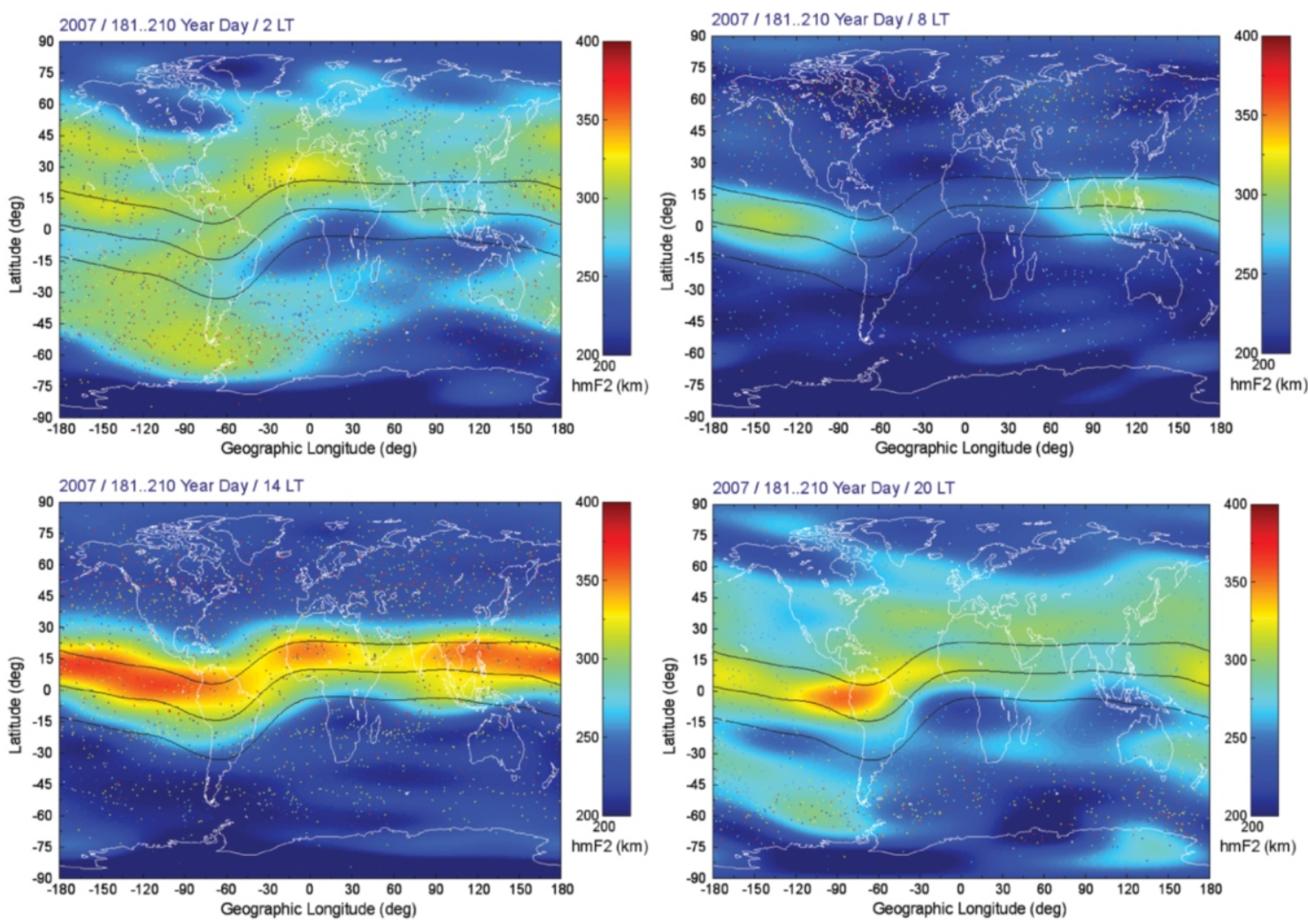

Fig. 11. The 2007 August $h m F 2$ numerical maps at 0200, 0800, 1400, and 2000 LT are assigned to the top-left, top-right, bottom-left, and bottom-right images, respectively. 


\section{CONCLUSIONS}

We conclude here that a physically appealing representation of $f_{o} F 2$ and $h m F 2$ is possible using GPS RO data. An advantage is data compilation that includes oceans and the southern hemisphere, where fewer ionosonde stations are positioned. Mapping analysis can be performed in different coordinate systems. We choose as our coordinate functions a particular set of the spherical surface harmonics $U_{n m}(\theta, \phi)$ and $V_{n m}(\theta, \phi)$ in the first three orders of $m=0,1$, and 2 only, but allow an optimum degree for each order to separate noise from real longitudinal variation. For the optimum separation of noise, we adopt a criterion whereby optimum cutoff is to search for a tendency to level off the absolute amplitude. Making use of the distributions of RO observations, we have computed the standard deviations of the $f_{O} F 2$ medians, too. In periods of low geomagnetic activity within the GPS/MET mission, $f_{0} F 2$ values determined by numerical mapping have smaller residuals in the middle than at the beginning (or end) of years. Furthermore, the new GPS RO mission of FS3/COSMIC could provide twelve times power of magnification for RO observations to determine more dense regions and the global distribution of $f o F 2$ and $h m F 2$ in numerical mapping. Calculations of average noise in $f o F 2$ and $h m F 2$ medians made over a period of several years is found to be closely correlated with geophysical activity. These investigations give very valuable information on large regions of the world, especially over seas where no dense networks for ionospheric observations exist.

Acknowledgements This work has been supported by Projects 96-NSPO(B)-SP-FA07-02-L and NSC96-2111M008-008-012.

\section{REFERENCES}

Bilitza, D., 2001: International Reference Ionosphere 2000. Radio Sci., 36, 261-275, doi: 10.1029/2000RS002432. [Link]

Davis, H. F., 1989: Fourier Series and Orthogonal Functions, Dover Publications, Inc., New York.

Dudeney, J. R., 1983: The accuracy of simple methods for determining the height of the maximum electron concentration of the F2-layer from scaled ionospheric characteristics. J. Atmos. Terr. Phys., 45, 629-640, doi: 10.1016/ S0021-9169(83)80080-4. [Link]

Garcia-Fernandez, M., M. Hernandez-Pajares, M. Juan, and J. Sanz, 2003: Improvement of ionospheric electron density estimation with GPSMET occultations using Abel inversion and VTEC information. J. Geophys. Res., 108, 13381344, doi: 10.1029/2003JA009952. [Link]

Hajj, G. A. and L. J. Romans, 1998: Ionospheric electron density profiles obtained with the Global Positioning System: Results from the GPS/MET experiment. Radio Sci., 33, 175-190, doi: 10.1029/97RS03183. [Link]

Hajj, G. A., L. C. Lee, X. Pi, L. J. Romans, W. S. Schreiner, P. R.
Straus, and C. Wang, 2000: COSMIC GPS ionospheric sensing and space weather. Terr. Atmos. Ocean. Sci., 11, 235-272.

Hanson, W. B. and R. J. Moffett, 1966: Ionization transport effects in the equatorial F region. J. Geophys. Res., 71, 5559-5572.

Hernández-Pajares, M., J. M. Juan, and J. Sanz, 2000: Improving the Abel inversion by adding ground GPS data to LEO radio occultations in ionospheric sounding. Geophys. Res. Lett., 27, 2743-2746, doi: 10.1029/2000GL000032. [Link]

Hocke, K. and K. Igarashi, 2002a: Structure of the Earth's lower ionosphere observed by GPS/MET radio occultation. $J$. Geophys. Res., 107, 1057, doi: 10.1029/2001JA900158. [Link]

Hocke, K. and K. Igarashi, 2002b: Electron density in the F region derived from GPS/MET radio occultation data and comparison with IRI. Earth Planets Space, 54, 947-954.

Kursinski, E. R., G. A. Hajj, J. T. Schofield, R. P. Linfield, and K. R. Hardy, 1997: Observing Earth's atmosphere with radio occultation measurements using the Global Positioning System. J. Geophys. Res., 102, 23429-23465, doi: 10.1029/97JD01569. [Link]

Rocken, C., R. Anthes, M. Exner, D. Hunt, S. Sokolovskiy, R. Ware, M. Gorbunov, W. Schreiner, D. Feng, B. Herman, Y.-H. Kuo, and X. Zou, 1997: Analysis and validation of GPS/MET data in the neutral atmosphere. J. Geophys. Res., 102, 29849-29866, doi: 10.1029/97JD02400. [Link]

Rush, C. M., M. PoKempner, D. N. Anderson, J. Perry, F. G. Stewart, and R. Reasoner, 1984: Maps of $f_{0} F_{2}$ derived from observations and theoretical data. Radio Sci., 19, 1083-1097, doi: 10.1029/RS019i004p01083. [Link]

Schreiner, W. S., S. V. Sokolovskiy, C. Rocken, and D. C. Hunt, 1999: Analysis and validation of GPS/MET radio occultation data in the ionosphere. Radio Sci., 34, 949-966, doi: 10.1029/1999RS900034. [Link]

Straus, P. R., 1999: Correcting GPS occultation measurements for ionospheric horizontal gradients. Proc. of Iono. Effect Symp., Alexandria, VA, June.

Tsai, L. C. and W. H. Tsai, 2004: Improvement of GPS/MET ionospheric profiling and validation with Chung-Li ionosonde measurements and the IRI. Terr. Atmos. Ocean. Sci., 15, 589-607.

Tsai, L. C., W. H. Tsai, W. S. Schreiner, F. T. Berkey, and J. Y. Liu, 2001: Comparisons of GPS/MET retrieved ionospheric electron density and ground based ionosonde data. Earth Planets Space, 53, 193-205.

Tsai, L. C., C. H. Liu, and T. Y. Hsiao, 2009: Profiling of ionospheric electron density based on FormoSat-3/COSMIC data: Results from the intense observation period experiment. Terr. Atmos. Ocean. Sci., 20, 181-191, doi: 10.3319/ TAO.2007.12.19.01(F3C). [Link]

URSI, 1987: Report of URSI Working Group G. 5 on mapping of characteristics at the peak of the F2 layer, Information Bulletin No. 243, Brussels, 93-96.

Ware, R., M. Exner, D. Feng, M. Gorbunov, K. Hardy, B. 
Herman, Y. Kuo, T. Meehan, W. Melbourne, C. Rocken, W. Schreiner, S. Sokolovskiy, F. Solheim, X. Zou, R. Anthes, S. Businger, and K. Trenberth, 1996: GPS sounding of the atmosphere from low Earth orbit: Preliminary results. Bull. Amer. Meteor. Soc., 77, 19-40, doi: 10.1175/
1520-0477(1996)077<0019:GSOTAF $>2.0 . C O ; 2$. [Link]

Zou, X., Y.-H. Kuo, and Y.-R. Guo, 1995: Assimilation of atmospheric radio refractivity using a nonhydrostatic adjoint model. Mon. Wea. Rev., 123, 2229-2249, doi: 10.1175/ 1520-0493(1995)123<2229:AOARRU>2.0.CO;2. [Link] 